\title{
A principal components analysis of factors associated with successful implementation of an LVAD decision support tool
}

\author{
Kristin M. Kostick ${ }^{1 *} \mathbb{D}$, Meredith Trejo ${ }^{1}$, Arvind Bhimaraj ${ }^{2}$, Andrew Civitello ${ }^{3}$, Jonathan Grinstein ${ }^{4}$, \\ Douglas Horstmanshof ${ }^{5}$, Ulrich P. Jorde ${ }^{6}$, Matthias Loebe7, Mandeep R. Mehra ${ }^{8}$, Nasir Z. Sulemanjee ${ }^{9}$, \\ Vinay Thohan ${ }^{10}$, Barry H. Trachtenberg ${ }^{2}$, Nir Uriel ${ }^{11}$, Robert J. Volk ${ }^{12}$, Jerry D. Estep ${ }^{13}$ and J. S. Blumenthal-Barby
}

\begin{abstract}
Background: A central goal among researchers and policy makers seeking to implement clinical interventions is to identify key facilitators and barriers that contribute to implementation success. Despite calls from a number of scholars, empirical insights into the complex structural and cultural predictors of why decision aids (DAs) become routinely embedded in health care settings remains limited and highly variable across implementation contexts.

Methods: We examined associations between "reach", a widely used indicator (from the RE-AIM model) of implementation success, and multi-level site characteristics of nine LVAD clinics engaged over 18 months in implementation and dissemination of a decision aid for left ventricular assist device (LVAD) treatment. Based on data collected from nurse coordinators, we explored factors at the level of the organization (e.g. patient volume), patient population (e.g. health literacy; average sickness level), clinician characteristics (e.g. attitudes towards decision aid; readiness for change) and process (how the aid was administered). We generated descriptive statistics for each site and calculated zero-order correlations (Pearson's $r$ ) between all multi-level site variables including cumulative reach at 12 months and 18 months for all sites. We used principal components analysis (PCA) to examine any latent factors governing relationships between and among all site characteristics, including reach.

Results: We observed strongest inclines in reach of our decision aid across the first year, with uptake fluctuating over the second year. Average reach across sites was $63 \%(s . d .=19.56)$ at 12 months and $66 \%(s . d .=19.39)$ at 18 months. Our PCA revealed that site characteristics positively associated with reach on two distinct dimensions, including a first dimension reflecting greater organizational infrastructure and standardization (characteristic of larger, more established clinics) and a second dimension reflecting positive attitudinal orientations, specifically, openness and capacity to give and receive decision support among coordinators and patients.

Conclusions: Successful implementation plans should incorporate specific efforts to promote supportive and mutually informative interactions between clinical staff members and to institute systematic and standardized protocols to enhance the availability, convenience and salience of intervention tool in routine practice. Further research is needed to understand whether "core predictors" of success vary across different intervention types.
\end{abstract}

\footnotetext{
*Correspondence: kristin.kostick@bcm.edu

${ }^{1}$ Center for Medical Ethics and Health Policy, Baylor College of Medicine,

One Baylor Plaza MC: 420, Houston, TX 77030, USA

Full list of author information is available at the end of the article
} original author(s) and the source, provide a link to the Creative Commons licence, and indicate if changes were made. The images or other third party material in this article are included in the article's Creative Commons licence, unless indicated otherwise in a credit line to the material. If material is not included in the article's Creative Commons licence and your intended use is not permitted by statutory regulation or exceeds the permitted use, you will need to obtain permission directly from the copyright holder. To view a copy of this licence, visit http://creativecommons.org/licenses/by/4.0/. The Creative Commons Public Domain Dedication waiver (http://creativeco mmons.org/publicdomain/zero/1.0/) applies to the data made available in this article, unless otherwise stated in a credit line to the data. 
Keywords: Implementation success, Facilitators and barriers, Decision support intervention, Principal components analysis

\section{Background}

A central goal among researchers and policy makers seeking to implement clinical interventions is to identify facilitators and barriers that contribute to implementation success. These factors often operate across multiple levels, including at the site-level (e.g. patient volume) [1], staff-level (e.g. turnover, motivation and buy-in) [2] and patient-level (e.g. patient health and literacy, language barriers) [2, 3]. Understanding how these diverse factors interact to impact on implementation outcomes is one of the primary challenges to designing effective implementation strategies, and the raison d'etre for theoretical frameworks like the Behavior Change Wheel, the Theoretical Domains Framework, and similar models that seek to characterize interventions and link them to specific target behaviors [4-6]. However, while conceptual models such as these can be useful for implementation planning, what researchers continue to lack are realworld, empirical examinations of site "profiles" that map onto implementation outcomes of varying success. What set of features facilitate a clinical intervention, such as an evidence-based decision aid, to be successfully implemented and sustained at some sites and not others?

Which factors are associated with successful implementation may indeed vary according to the nature of an intervention. However, few empirical data yet exist to evaluate this claim, or to systematically examine to what degree site characteristics vary while still contributing to implementation successes. Likewise, little is known about whether certain site characteristics predicting success regularly overlap to form a constellation of "core implementation success predictors" applicable across interventions. These gaps constitute a continuing impediment to understanding factors contributing to implementation success and its broader impacts on shared decision making (SDM) [7].

The importance and enduring challenge of identifying factors contributing to the successful "reach" of an intervention must be understood within a larger context of the "implementation gap" described by Gayer et al. [8] in the uptake of evidence-based decision aids compared to their use in real-world settings. Decision aids (DAs) are tools designed to increase patient knowledge about risks and benefits of treatment alternatives, help patients clarify what is important to them in making a decision, and to increase patient engagement in shared decision making. The utility of DAs rests on over two decades of efficacy research [9], yet their uptake in practice continues to be limited [10]. A systematic review by Elwyn et al. (2013) attributed this implementation gap to indifference on the part of health care professionals, stemming from a lack of confidence in the content of decision support interventions and concern about disruption to established workflows [7]. More recently, Scholl et al. (2018) recommended more closely examining how organizational-level factors (leaders, culture, resources, priorities, team dynamics and workflows) and system-level characteristics (policies, clinical guidelines, incentives, culture, education and licensing) and their interactions influence implementation success [1]. Despite calls from a number of scholars, empirical insights into the complex structural and cultural predictors of why DAs become routinely embedded in health care settings remains limited and highly variable across implementation contexts [7, 11-15]. Further, few studies explicitly compare feature profiles of different implementation sites with varying levels of success in order to gain insight into what site attributes, practices or attitudinal orientations facilitate implementation success [16, 17]. In this paper we offer results from a 9-site project to disseminate and implement a validated DA for patients considering left ventricular assist device (LVAD) therapy for advanced heart failure [18-20].

\section{Description of implementation project}

We tested our LVAD DA in a multisite randomized control trial (RCT) between 2015 and 2017 and found it significantly increased LVAD knowledge [21]. Once validated, we sought to implement the DA into clinical practice. The goals of our implementation project were to (1) Build capacity with key clinicians (physicians and LVAD nurse coordinators) to implement our LVAD DA through an initial "Capacity Building Webinar" and reinforcement sessions [22]; (2) Collaborate with "physician champions" to support LVAD nurse coordinators in their efforts to implement the DA during patient education and to use the DA themselves; and (3) Provide ongoing support to LVAD coordinators to facilitate development of sustainable practices for long-term DA use in their programs. The implementation setting includes nine U.S. hospitals, including five that participated in our original RCT of the DA and four that had no prior experience with the LVAD DA [20]. None of the nine sites were actively using the DA at the beginning of the dissemination and implementation (D\&I) project due to staff turnover since the RCT. 
We engaged LVAD nurse coordinators as primary staff to disseminate and review the DA with patients. Coordinators generally provide LVAD education and have frequent contact with patient candidates during LVAD evaluation.

Evaluation of dissemination and implementation (D\&I) progress and success is based on the RE-AIM framework (reach, effectiveness, adoption, implementation, maintenance) [23], tracking outcomes using a 10-item "Implementation Tracking Sheet" (ITS) completed for each DA use which measures fidelity to the intended use of our DA in the context of SDM. Among the variables in the REAIM framework, reach (percentage of eligible patients receiving the intervention) is our primary indicator and a widely recognized outcome measure for gauging the success of implementation.

In an attempt to better understand which site features contribute to greater reach we conducted brief surveys with LVAD physicians and coordinators to identify objective site characteristics (e.g. patient volume, patient sickness level) and subjective orientations (e.g. clinician readiness for change; coordinator/clinician satisfaction with the DA; perceived integration with existing educational materials) with the potential to impact implementation success).

\section{Methods \\ Participants and variable selection}

We conducted a brief online survey with LVAD coordinators (December 2019-March 2020) across 9 clinical sites currently participating in our 2-year implementation project, selected on the basis of our previous relationships with these clinics while developing and testing our DA. The purpose of the survey was to collect information from healthcare professionals (reporting to the best of their knowledge) at participating sites about characteristics of their clinical sites and patient populations, as well as their attitudes towards and use of the DA. The survey was administered using Microsoft Forms once per respondent over months $14-17$ in order to capture site dynamics representative of and relevant to both of the cross-sectional time points (months 12 and 18) at which reach was assessed. Participants were notified in advance that they would be sent a $\$ 75$ gift card upon completion of the survey. While individual respondents remained anonymous, their degree of anonymity was limited by

Table 1 Clinical site variables explored

\begin{tabular}{|c|c|c|}
\hline Variable type & Variable construct & Question item \\
\hline \multirow[t]{2}{*}{ Organizational } & Pt Volume: Implants & In the last year approximately how many LVAD implants were placed at your hospital? \\
\hline & Pt Volume: Evaluations & $\begin{array}{l}\text { In the last year, approximately how much new evaluations for LVAD did your hospital } \\
\text { have? }\end{array}$ \\
\hline \multirow[t]{3}{*}{ Patient } & Pt Sickness Level & $\begin{array}{l}\text { Compared to other hospitals, do you think the patients your hospital evaluates are more } \\
\text { or less sick? }\end{array}$ \\
\hline & Pt Health Literacy & $\begin{array}{l}\text { Compared to other hospitals, do you think the health literacy of patients evaluated for } \\
\text { LVAD is greater or lesser? }\end{array}$ \\
\hline & Pt Language Barriers & How frequently are you unable to use the LVAD decision aid due to language barriers? \\
\hline \multirow[t]{7}{*}{ Clinician/Staff } & Experience as LVAD Pt Educator & For how many years have you worked as an LVAD coordinator/educator/engineer? \\
\hline & Time Spent on LVAD Pt Education & What portion of your time is spent on LVAD Education? \\
\hline & Use of DA Compared to Other Clinicians & $\begin{array}{l}\text { Compared to other staff on your team, do you use the decision aid more, less, or about the } \\
\text { same? }\end{array}$ \\
\hline & Coordinator-Clinician Interaction & $\begin{array}{l}\text { How frequently do you talk with the physicians at your hospital regarding a patient's LVAD } \\
\text { evaluation? }\end{array}$ \\
\hline & Satisfaction with DA & How satisfied are you with the LVAD decision aid as a resource for patient education? \\
\hline & Satisfaction with Standard Education & $\begin{array}{l}\text { Before you started using the LVAD decision aid, how satisfied were you with the LVAD } \\
\text { education materials provided by your hospital? }\end{array}$ \\
\hline & Readiness for Change (ORIC) & $\begin{array}{l}\text { Respondent's score on the Organizational Readiness for Instituting Change scale. } \\
\text { Organizational readiness is broadly defined by members' psychological and behavioral } \\
\text { preparedness to implement organization change }\end{array}$ \\
\hline \multirow[t]{2}{*}{ Process } & Standardization of DA Use & $\begin{array}{l}\text { Do you give patients the LVAD decision aid at the same point during education and evalu- } \\
\text { ation for candidacy? }\end{array}$ \\
\hline & Integration of DA with Standard Education & $\begin{array}{l}\text { How do you use the LVAD decision aid with your hospital's existing (before decision aid) } \\
\text { education materials? }\end{array}$ \\
\hline \multirow[t]{2}{*}{ Outcome } & Reach: 12 months & Proportion of eligible individuals who received the DA by 12 months \\
\hline & Reach: 18 months & Proportion of eligible individuals who received the DA by 18 months \\
\hline
\end{tabular}


their identification of their clinical site, which was needed to match their responses to reach performance.

Variables explored in the survey are reported in Table 1 and include site characteristics at the level of the organization (volume of annual patient LVAD evaluations and LVAD implants); patient (perceived average patient sickness level; perceived average health literacy and language barriers); clinician (experience; workload; degree of coordinator interaction with physicians; attitudes towards and use of DA versus standard education; readiness for change; distribution of DA administration responsibilities across clinical staff); and process (e.g. standardization of DA administration; integration of DA with existing educational materials).

We used "reach" from the widely used RE-AIM model as our primary outcome measure of implementation [23], defined as the proportion of eligible patients (in our case, patients under clinical evaluation for LVAD therapy) who received a DA before completing clinical evaluation and/or making a treatment choice. The decision to include the range of variables outlined above (e.g. organizational level, patient level, etc.) is based on a literature review of potential factors influencing reach [1, 17, 24-26]. For each clinical site, reach was calculated at 12 and 18 months into the implementation process. Each site submitted an ITS for each patient receiving the LVAD DA which is how we tracked the number of patients receiving a DA. Sites submitted the ITS immediately after using the DA with a patient or in aggregate for all monthly patients at the end of the month. Each month sites also reported their total number of eligible patients (patients under LVAD evaluation). The number of ITSs received was divided by the total number of evaluations to determine reach.

Because we did not want to impose an arbitrary threshold by which we considered a clinical site to have achieved implementation "success," we sought instead to understand reach scores in a relative rather than absolute way and thus categorized each site in relation to the mean across sites at each time point (see "Results" section). Specifically, reach was categorized as high ( $\geq$ one standard deviation above the reach mean at each time point), medium (between one standard deviation above and below the reach mean) or low (below one standard deviation below the mean). For example, if mean reach at 12 months was $63 \%$ and the standard deviation was $19 \%$, "low" reach would be characterized as any reach score at or below $44 \%$ (i.e. less than one standard deviation below the mean, or $63-19=44 \%$ ). We also categorized reach this way to better correspond to the structure of our survey responses (e.g. high, medium/neutral, low).

We measured respondents' readiness to implement change, broadly defined by individuals' psychological and behavioral preparedness to implement organizational change, using the widely endorsed Organizational Readiness for Implementing Change (ORIC) scale [27]. This scale examines the degree to which individuals are likely to initiate change, exert greater effort, and exhibit greater persistence in implementing an intervention. Responses were scored according to the original authors' instructions, with positive scores indicating greater readiness, and sites characterized as above (high) or below (low) or between (medium) one standard deviation from the mean of 51.5 (out of 60). These and all other variables are valenced with high scores indicating greater/more of the variable unit.

\section{Analysis}

Surveys and reach data were recorded and analyzed in Excel (XLStat 2020.3.1.4). We generated descriptive statistics for each site, with survey scores from multiple clinicians from a single clinic collapsed by averaging and rounding to the nearest integer to form one score per variable per clinic. We calculated zero-order correlations (Pearson's $r$ ) between all multi-level site variables including cumulative reach at 12 months and 18 months for all sites, and principal components analysis (PCA) to examine any latent factors governing relationships between and among all site characteristics, including reach.

\section{Results}

Implementation success outcomes (reach)

We observed strongest inclines in reach of our decision aid across the first year, with the frequency of uptake fluctuating over the second year. Figure 1 presents changes in average reach levels across clinical sites over a period of 18 months (November 2019-April 2020) following initial implementation (two months consisting of orientation and startup) in September-October 2018. Average reach across sites was $63 \%$ (s.d. $=19.56)$ at 12 months and $66 \%$ $($ s.d. $=19.39)$ at 18 months. Three out of nine sites (33\%) reported high reach at both time points. Peaks in average reach were highest in months 6-8 (February-April 2019) and at the end of 18 months (April 2020). Lower reach levels were observed over the summer months as well as in October and December of 2019 (leading up to holidays) and in March 2020 (coinciding with rising cases of COVID-19 in the United States).

\section{Organizational characteristics of clinical sites and healthcare professionals}

Descriptive site characteristics for all nine sites are presented in Table 2. Average reach scores at both 12- and 18-month time points only included eight sites, as one site did not achieve sufficient implementation startup (i.e. the site was unable to consistently provide reach data). 


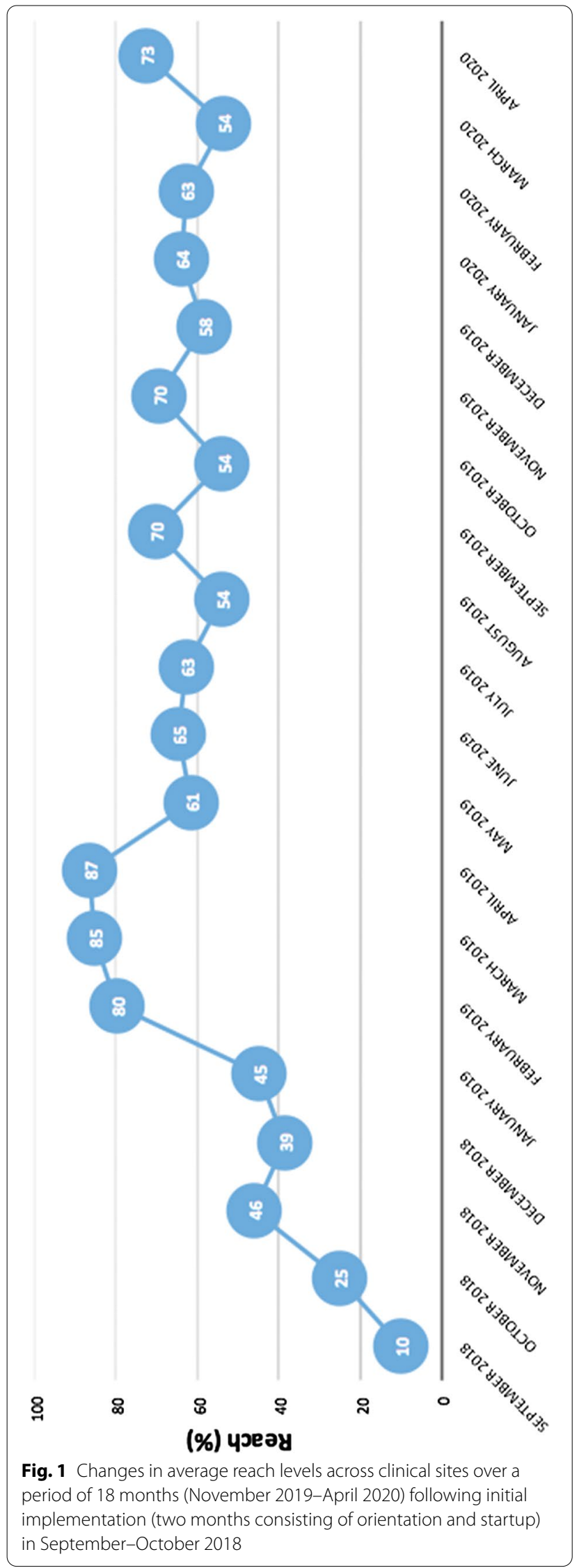

Most sites (44\% and 56\%, respectively) reported a moderate patient volume of 31-50 implants per year and a high $(\mathrm{n}=>100)$ volume of evaluations. Most (78\%) respondents characterized their patient populations as "more sick" compared to those at other LVAD programs, and most ( $78 \%$ and $56 \%$, respectively) said their patients had about the same literacy and English language proficiency as other sites. Over half of respondents (56\%) reported 0-4 years of experience with LVAD patient education and $67 \%$ said that their work duties are devoted only in part ("some of my time" versus "all" or "little") to patient education. Other duties included administrative responsibilities and clinical care for other types of heart failure patients. Over half (56\%) reported using the DA about the "same amount" as other clinical staff involved with LVAD education.

\section{Clinician attitudes and readiness for implementation}

Over three quarters $(78 \%)$ of respondents reported feeling somewhat or very satisfied with the DA, while only $56 \%$ said the same about standard education materials. Just under half (44\%) demonstrated high readiness for change based on responses to the ORIC scale, with $22 \%$ reporting low readiness.

\section{Process-related barriers/facilitators}

A majority (89\%) of sites reported a high level of standardization in DA use. Almost half (44\%) of sites reported moderate integration of the DA with existing standard education materials (i.e. using them about the same amount) as opposed to using the DA more (33\%) or less (22\%) than standard education materials.

\section{Associations between site characteristics and reach}

Table 3 presents zero-order correlations between site characteristic variables, with significant correlations $(\mathrm{p} \leq 0.05)$ bolded. We observed a significant negative correlation between clinician experience and the proportion of their duties or time spent on patient LVAD education $(\mathrm{r}=-0.74 ; \mathrm{p} \leq 0.05)$. We also found significant positive correlations between the level of coordinator-physician interaction and a clinician's use of the DA compared to other staff $(\mathrm{r}=0.71 ; \mathrm{p} \leq 0.05)$; between the perceived health literacy of a patient population and coordinator/ clinician satisfaction with standard education materials and procedures $(\mathrm{r}=0.88 ; \mathrm{p} \leq 0.05)$; standardization of DA use within a clinical site and a clinician's more frequent use of the DA compared to other clinicians $(r=0.88$; $\mathrm{p} \leq 0.0571$ ); and between a site's number of LVAD patient evaluations and implants $(\mathrm{r}=0.86 ; \mathrm{p} \leq 0.05)$. We also found a perfect correlation between overall average reach at 12 months and at 18 months $(\mathrm{r}=1.0, \mathrm{p} \leq 0.05)$, though 
Table 2 Descriptive statistics for participating clinical sites $(n=9)$

\begin{tabular}{|c|c|c|}
\hline Variable type & Characteristic & Percentage \\
\hline \multicolumn{3}{|c|}{ Organizational Variables } \\
\hline \multicolumn{3}{|c|}{ Patient Volume: Implants } \\
\hline & $11-30$ implants & $33 \%$ \\
\hline & $31-50$ implants & $44 \%$ \\
\hline & $>50$ implants & $22 \%$ \\
\hline \multicolumn{3}{|c|}{ Patient Volume: Evaluations } \\
\hline & $36-50$ evaluations & $33 \%$ \\
\hline & $51-100$ evaluations & $11 \%$ \\
\hline & $>100$ evaluations & $56 \%$ \\
\hline \multicolumn{3}{|c|}{ Patient Variables } \\
\hline \multicolumn{3}{|c|}{ Patient sickness level } \\
\hline & Less sick & $0 \%$ \\
\hline & About the same & $22 \%$ \\
\hline & More sick & $78 \%$ \\
\hline \multicolumn{3}{|c|}{ Patient health literacy } \\
\hline & Lower health literacy & $22 \%$ \\
\hline & About the same health literacy & $78 \%$ \\
\hline & Higher health literacy & $0 \%$ \\
\hline \multicolumn{3}{|c|}{ Patient language barriers } \\
\hline & Rarely & $44 \%$ \\
\hline & Sometimes & $56 \%$ \\
\hline & Often & $0 \%$ \\
\hline \multicolumn{3}{|c|}{ Clinician/Staff Variables } \\
\hline \multicolumn{3}{|c|}{ Years of experience as VAD coordinator } \\
\hline & $0-4$ years & $56 \%$ \\
\hline & $5-9$ years & $33 \%$ \\
\hline & $\geq 10$ years & $11 \%$ \\
\hline \multicolumn{3}{|c|}{ Average time spent on LVAD patient education } \\
\hline & Little of my time & $11 \%$ \\
\hline & Some of my time & $67 \%$ \\
\hline & All of my time & $22 \%$ \\
\hline \multicolumn{3}{|c|}{ Use of decision aid compared to other clinical staff } \\
\hline & Less than others & $11 \%$ \\
\hline & Same amount as others & $56 \%$ \\
\hline & More than others or only user & $33 \%$ \\
\hline \multicolumn{3}{|c|}{ Level of LVAD coordinator and physician interaction } \\
\hline & Rarely & $0 \%$ \\
\hline & Sometimes & $11 \%$ \\
\hline & Frequently or always & $89 \%$ \\
\hline \multicolumn{3}{|c|}{ Satisfaction with decision aid } \\
\hline & Somewhat or very dissatisfied & $0 \%$ \\
\hline & Neutral & $22 \%$ \\
\hline & Somewhat or very satisfied & $78 \%$ \\
\hline \multicolumn{3}{|c|}{ Satisfaction with standard education materials } \\
\hline & Somewhat or very dissatisfied & $22 \%$ \\
\hline & Neutral & $22 \%$ \\
\hline & Somewhat or very satisfied & $56 \%$ \\
\hline \multicolumn{3}{|c|}{ Readiness for Change (ORIC) } \\
\hline & Less ready & $22 \%$ \\
\hline
\end{tabular}


Table 2 (continued)

\begin{tabular}{|c|c|c|}
\hline Variable type & Characteristic & Percentage \\
\hline & Moderately ready & $33 \%$ \\
\hline & Very ready & $44 \%$ \\
\hline \multicolumn{3}{|l|}{ Process } \\
\hline \multicolumn{3}{|c|}{ Standardization of decision aid (DA) use } \\
\hline & Rarely & $0 \%$ \\
\hline & Sometimes & $11 \%$ \\
\hline & Frequently & $89 \%$ \\
\hline \multicolumn{3}{|c|}{ Integration of decision aid (DA) with standard education (SE) materials } \\
\hline & Use SE more than the DA & $22 \%$ \\
\hline & Use DA and SE about same & $44 \%$ \\
\hline & Use DA exclusively or more than SE & $33 \%$ \\
\hline \multicolumn{3}{|l|}{ Outcome } \\
\hline \multicolumn{3}{|c|}{ Reach: 12 months ${ }^{*} \wedge$} \\
\hline & Sites with low reach $(\leq \bar{x})$ & $22 \%$ \\
\hline & $\begin{array}{l}\text { Sites with medium reach } \\
44 \%-82 \% \text { (between } 1 \text { s.d } \\
\text { above/below } \bar{x} \text { ) }\end{array}$ & $44 \%$ \\
\hline & Sites with high reach $\geq \bar{x}$ & $33 \%$ \\
\hline \multicolumn{3}{|c|}{ Reach: 18 months $^{* * \wedge}$} \\
\hline & Sites with low reach $(\leq \bar{x})$ & $22 \%$ \\
\hline & $\begin{array}{l}\text { Sites with medium reach } \\
46 \%-84 \% \text { (between } 1 \mathrm{~s} . d \\
\text { above/below } \bar{x} \text { ) }\end{array}$ & $44 \%$ \\
\hline & Sites with high reach $\geq \bar{x}$ & $33 \%$ \\
\hline
\end{tabular}

${ }^{*} \mathrm{X}=63 \% ;$ s.d. $=19 \%$

${ }^{* *} \mathrm{x}=65 \%$; s.d. $=19 \%$

$\wedge$ Does not include reach from one site who did not achieve start-up

May not sum to $100 \%$ due to rounding

Highest percentage values are bolded for each variable

Table 3 Site characteristic (Pearson's r) correlations across 9 LVAD clinics

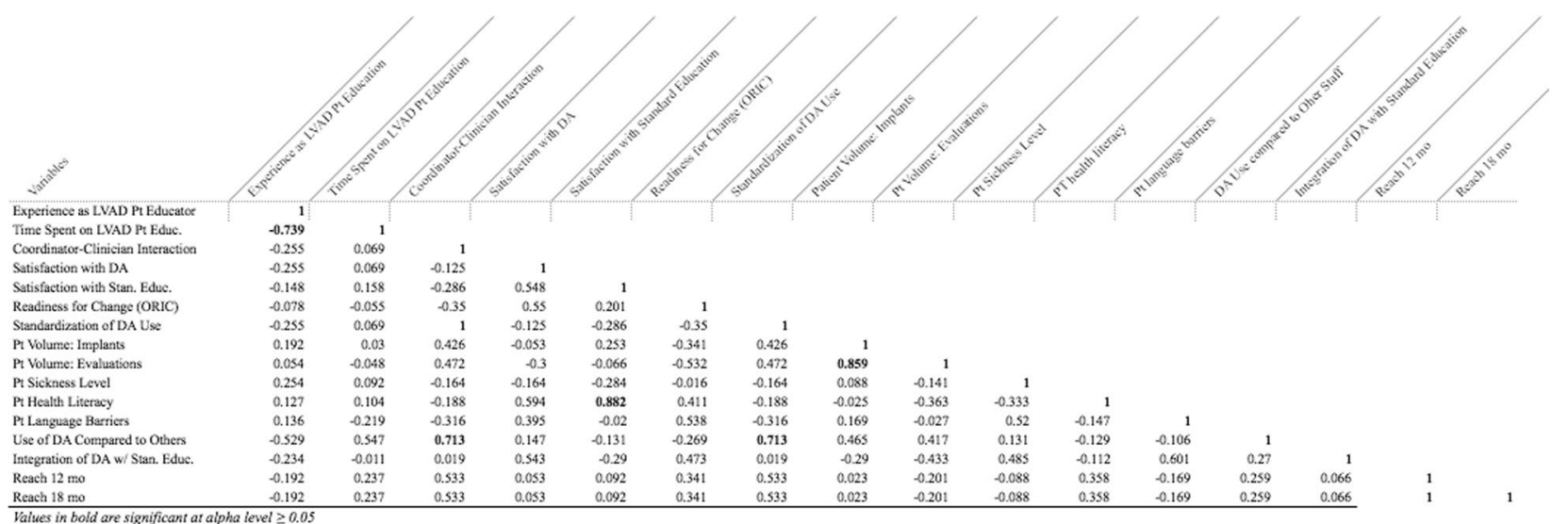

individual sites varied in the consistency of their reach over these two time points.

A principal components analysis revealed a cluster of site characteristics positively associated with reach on two distinct dimensions, which we interpreted to reflect a structural/organizational dimension (F2) and an attitudinal dimension (F1). Reach was particularly central on factor two (F2), where we observed high positive 
Table 4 Principal component analysis: eigenvalues for factors $1-5$

\begin{tabular}{lccccc}
\hline & $F 1$ & $F 2$ & $F 3$ & $F 4$ & $F 5$ \\
\hline Eigenvalue & 4.30 & 3.45 & 2.59 & 1.98 & 1.73 \\
Variability (\%) & 26.9 & 21.6 & 16.2 & 12.4 & 10.8 \\
Cumulative \% & 26.9 & 48.4 & 64.6 & 77.0 & 87.8 \\
\hline
\end{tabular}

associations (i.e. factor loadings $\geq 0.66$ ) with reach for variables including perceived patient health literacy, clinician/coordinator satisfaction with the DA, and readiness for change (see Table 7). Another dimension (F1) accounted for roughly equivalent variance (see Table 4) and showed high positive associations (factor loading $\geq 0.50$ ) for variables including level of coordinatorphysician interaction, standardization of DA use, use of DA compared to other staff, and high volume of patient evaluations and implants (see Table 5). These variables appeared together with reach at 12 and 18 months (factor loadings $=0.42)($ Table 6$)$.

The Kaiser-Meyer-Olkin co-efficient, which measures the proportion of variance among variables that might be due to common variance, was moderately low (0.38), indicating that, overall, site variables are only loosely related (are independent of one another), or that more

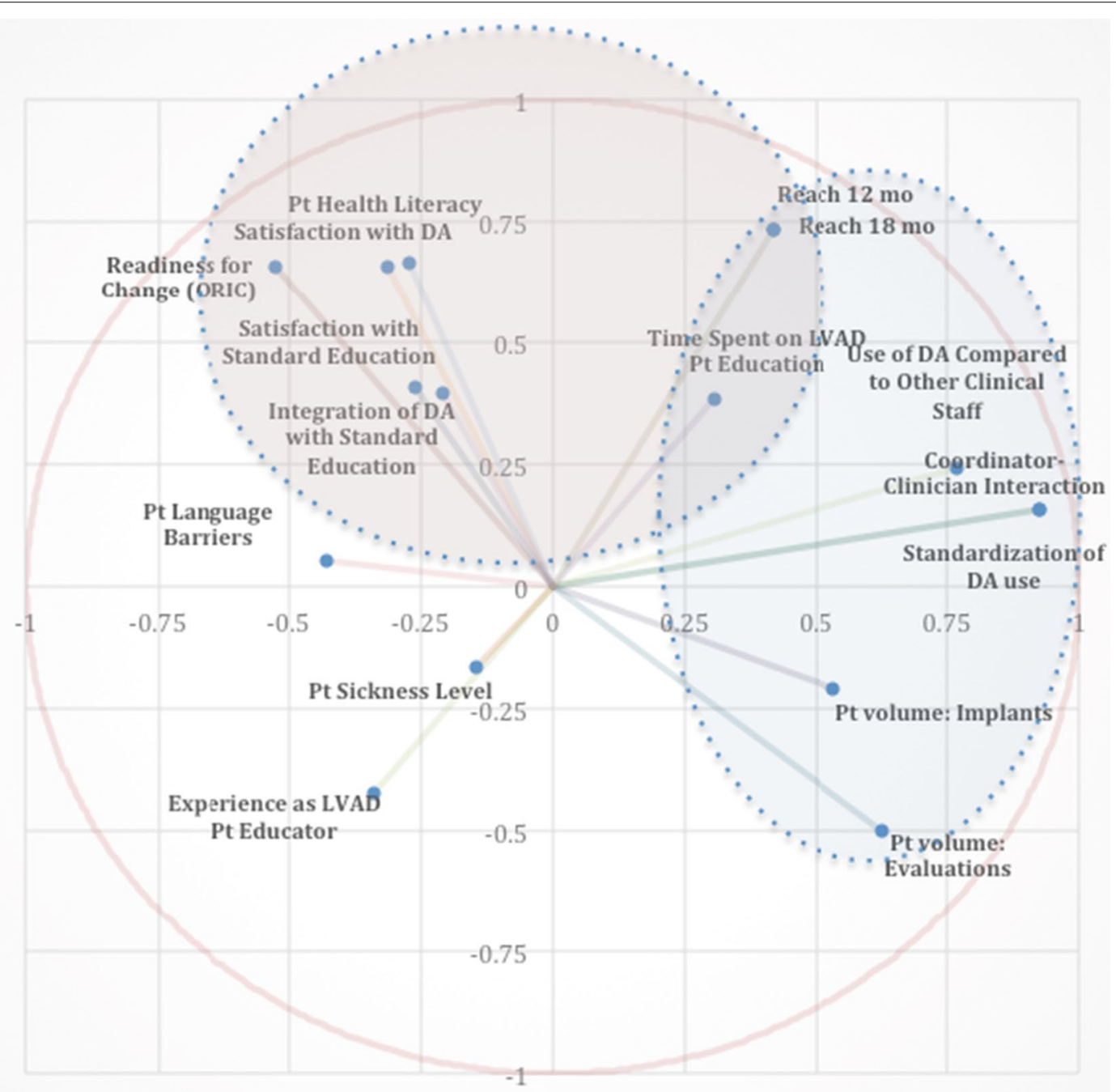

Variables on axes F1 X F2 $=\mathbf{4 8 . 4 4} \%$ variance

Variables Associated with High Reach on Factor 1 (Circle 1) and Factor 2

(Circle 2)

Fig. 2 Multidimensional scale of site characteristics in relation to reach 
Table 5 Variable factor loadings (F1-F5) and \% contributions ordered by F1 loadings

\begin{tabular}{lccccccccccc}
\hline & $F 1$ & $\%$ & $F 2$ & $\%$ & $F 3$ & $\%$ & $F 4$ & $\%$ & $F 5$ & $\%$ \\
\hline Coordinator-Clinician Interaction & $\mathbf{0 . 9 3}$ & $\mathbf{1 9 . 9}$ & 0.16 & 0.70 & 0.09 & 0.31 & 0.02 & 0.02 & -0.23 & 3.02 \\
Standardization of DA Use & $\mathbf{0 . 9 3}$ & $\mathbf{1 9 . 9}$ & 0.16 & 0.70 & 0.09 & 0.31 & 0.02 & 0.02 & -0.23 & 3.02 \\
Use of DA Compared to Other Clinical Staff & $\mathbf{0 . 7 7}$ & $\mathbf{1 3 . 7}$ & 0.24 & 1.67 & 0.32 & 4.00 & 0.27 & 3.72 & 0.32 & 6.09 \\
Pt volume: Evaluations & $\mathbf{0 . 6 3}$ & $\mathbf{9 . 0 9}$ & -0.50 & 7.28 & -0.15 & 0.90 & 0.50 & 12.45 & 0.05 & 0.13 \\
Pt volume: Implants & $\mathbf{0 . 5 3}$ & $\mathbf{6 . 5 9}$ & -0.21 & 1.26 & -0.17 & 1.16 & 0.77 & 29.81 & -0.08 & 0.36 \\
Reach 18 mo & $\mathbf{0 . 4 2}$ & $\mathbf{4 . 0 7}$ & 0.73 & 15.50 & -0.06 & 0.15 & -0.19 & 1.84 & -0.43 & 10.7 \\
Reach 12 mo & $\mathbf{0 . 4 2}$ & $\mathbf{4 . 0 7}$ & 0.73 & 15.50 & -0.06 & 0.15 & -0.19 & 1.84 & -0.43 & 10.7 \\
Time Spent on LVAD Pt Education & $\mathbf{0 . 3 0}$ & $\mathbf{2 . 1 5}$ & 0.38 & 4.27 & 0.07 & 0.20 & -0.11 & 0.59 & 0.71 & 28.7 \\
Pt Sickness Level & -0.14 & 0.48 & -0.16 & 0.78 & 0.66 & 16.9 & 0.20 & 2.00 & -0.15 & 1.29 \\
Integration of DA with Standard Education & -0.21 & 1.02 & 0.39 & 4.48 & 0.83 & 26.4 & 0.14 & 1.02 & -0.04 & 0.07 \\
Satisfaction with Standard Education & -0.26 & 1.60 & 0.41 & 4.83 & -0.68 & 17.7 & 0.48 & 11.7 & 0.17 & 1.69 \\
Pt Health Literacy & -0.27 & 1.73 & 0.67 & 12.81 & -0.61 & 14.4 & 0.22 & 2.45 & -0.01 & 0.01 \\
Satisfaction with DA & -0.31 & 2.30 & 0.66 & 12.49 & 0.06 & 0.13 & 0.48 & 11.7 & 0.23 & 3.18 \\
Experience as LVAD Pt Educator & -0.34 & 2.70 & -0.42 & 5.18 & -0.28 & 3.04 & 0.25 & 3.05 & -0.68 & 26.4 \\
Pt Language Barriers & -0.43 & 4.25 & 0.05 & 0.07 & 0.58 & 12.9 & 0.59 & 17.5 & -0.18 & 1.80 \\
Readiness for Change (ORIC) & -0.53 & 6.45 & 0.66 & 12.45 & 0.19 & 0.08 & 0.09 & 0.30 & 0.30 & 2.68 \\
\hline
\end{tabular}

Bold values represent positive factor loadings $\geq 0.30$ on Factors 1 and 2, respectively

Table 6 Variable factor loadings (F1-F5) and \% contributions ordered by F2 Loadings

\begin{tabular}{lcccccccccc}
\hline & $F 1$ & $\%$ & $F 2$ & $\%$ & $F 3$ & $\%$ & $F 4$ & $\%$ & $F 5$ & $\%$ \\
\hline Reach 18 mo & 0.42 & 4.07 & $\mathbf{0 . 7 3}$ & $\mathbf{1 5 . 5}$ & -0.06 & 0.15 & -0.19 & 1.84 & -0.43 & 10.7 \\
Reach 12 mo & 0.42 & 4.07 & $\mathbf{0 . 7 3}$ & $\mathbf{1 5 . 5 0}$ & -0.06 & 0.15 & -0.19 & 1.84 & -0.43 & 10.7 \\
Pt Health Literacy & -0.27 & 1.73 & $\mathbf{0 . 6 7}$ & $\mathbf{1 2 . 8}$ & -0.61 & 14.4 & 0.22 & 2.45 & -0.01 & 0.01 \\
Satisfaction with DA & -0.31 & 2.30 & $\mathbf{0 . 6 6}$ & $\mathbf{1 2 . 5}$ & 0.06 & 0.13 & 0.48 & 11.7 & 0.23 & 3.18 \\
Readiness for Change (ORIC) & -0.53 & 6.45 & $\mathbf{0 . 6 6}$ & $\mathbf{1 2 . 5}$ & 0.19 & 1.40 & 0.08 & 0.30 & -0.22 & 2.68 \\
Satisfaction with Standard Education & -0.26 & 1.60 & $\mathbf{0 . 4 1}$ & $\mathbf{4 . 8 3}$ & -0.68 & 17.7 & 0.48 & 11.7 & 0.17 & 1.69 \\
Integration of DA with Standard Education & -0.21 & 1.02 & $\mathbf{0 . 3 9}$ & $\mathbf{4 . 4 8}$ & 0.83 & 26.4 & 0.14 & 1.02 & -0.04 & 0.07 \\
Time Spent of LVAD Pt Education & 0.30 & 2.15 & $\mathbf{0 . 3 8}$ & $\mathbf{4 . 2 7}$ & 0.07 & 0.20 & -0.11 & 0.59 & 0.71 & 28.7 \\
Use of DA Compared to Other Clinical Staff & 0.77 & 13.7 & 0.24 & 1.67 & 0.32 & 4.00 & 0.27 & 3.72 & 0.32 & 6.09 \\
Coordinator-Clinician Interaction & 0.93 & 19.9 & 0.16 & 0.70 & 0.09 & 0.31 & 0.02 & 0.02 & -0.23 & 3.02 \\
Standardization of DA Use & 0.93 & 19.92 & 0.16 & 0.70 & 0.09 & 0.31 & 0.02 & 0.02 & -0.23 & 3.02 \\
Pt Language Barriers & 0.043 & 4.25 & 0.05 & 0.07 & 0.58 & 12.9 & 0.59 & 17.5 & -0.18 & 1.80 \\
Pt Sickness Level & -0.14 & 0.48 & -0.16 & 0.78 & 0.66 & 16.9 & 0.20 & 2.00 & -0.15 & 1.29 \\
Pt Volume: Implants & 0.53 & 6.59 & -0.21 & 1.26 & -0.17 & 1.16 & 0.77 & 29.8 & -0.08 & 0.36 \\
Experience as LVAD Pt Educator & -0.34 & 2.70 & -0.42 & 5.18 & -0.28 & 3.04 & 0.25 & 3.05 & -0.68 & 26.4 \\
Pt Volume: Evaluations & 0.63 & 9.09 & -0.50 & 7.28 & -0.15 & 0.90 & 0.50 & 12.5 & 0.05 & 0.13 \\
\hline
\end{tabular}

Bold values represent positive factor loadings $\geq 0.30$ on Factors 1 and 2, respectively

sites must be sampled in order to better understand how site variables are associated. Factors 1 and 2 pictured in the multidimensional scale in Fig. 2 account for $48 \%$ of the variance, leaving over $50 \%$ of the variance unexplained by the measured variables.

\section{Discussion}

Strongest inclines in uptake and reach of our decision aid occurred across the first year, with frequency of uptake fluctuating over the second year. That the largest of these fluctuations coincided with typical seasonal/holiday shifts (October-December 2019) as well as the sharp rise of the COVID-19 pandemic in the United Stated (March 2020), suggests that larger situational factors at the national or even global level can influence reach. At a more local level, however, we discovered key site characteristics that appear to have important associations with reach. These site characteristics form two distinct constellations of variables that are non-overlapping, apart from their pivotal associations with reach, suggesting there may be more than one effective pathway to implementation success. 
On one dimension, we found that reach is associated with site characteristics indicating greater organizational infrastructure and clinical standardization, variables typical of larger, more established clinics. Specifically, this constellation of traits includes greater volume of patient evaluations and implants, more frequent physician involvement with LVAD nurse coordinators, more highly specified role distribution (i.e. designated personnel for delivering patient education and decisional support), and greater standardization of patient education protocols.

Meanwhile, on a separate, second dimension, reach showed strong associations with a group of variables suggesting the importance of attitudinal orientation, including openness and capacity to give and receive decision support among coordinators and LVAD candidates, respectively. On this factor, high reach was associated with greater patient health literacy, greater coordinator/ clinician satisfaction with the DA, and greater readiness for change. Thus, a patient's ability to understand the health information conveyed during patient education, coupled with a coordinator's more positive attitudes toward the DA and a broader sentiment among other staff of readiness for improvements, may together facilitate greater uptake of decision support.

\section{The attitudinal dimension: pro-change attitudes and motivation}

An openness to provide and receive decision support has long been recognized as a crucial ingredient in the uptake of shared decision making tools $[1,15,28]$. Indeed, motivation of health professionals was found to be a top factor in a systematic review of barriers and facilitators of implementing SDM, including DAs, in practice [13]. However, generating "buy-in" to the theoretical and practical importance of providing decision support is one of the most formidable challenges to effective implementation. A number of documented implementation approaches center on providing education to clinicians and staff members [28-32]. However, the assumption that with knowledge comes motivation may be erroneous, and some researchers [33-35] have pointed out that education- or persuasion-based approaches may be ineffective if they do not also address individuals' diverse motivations for engaging with an intervention. Such approaches are likely to encounter resistance from clinical staff's default, habitual ways of thinking and behaving (sometimes called "bounded rationality") [36].

To address the need for fostering incentives that are internalized as positive, reflexive attitudes towards an intervention, we have outlined elsewhere a series of tools designed to positively affect clinicians' and staff members' motivations for using a DA [37]. This toolkit, called MINDSPACE, draws from robust insights in behavioral economics to offer empirically supported strategies for implementers seeking to initiate small attitudinal, emotional, or behavioral responses that can collectively help bring about lasting, positive behavioral change. One optimistic insight is that attitudes and orientations towards shared decision-making and positive change may be more easily altered than structural variables, such as patient volume or available time spent on patient education, and are likely to be less economically and logistically costly to modify [37]. For this reason, implementation scientists seeking to foster clinical characteristics associated with successful implementation of decision support tools may look towards strategies to generate staff interest in and motivation to use SDM tools. This recommendation is widely cited by an already sizable implementation science literature and empirically supported by our findings $[1,15,28]$.

\section{The structural dimension: organizational size, standardization and interaction}

A second implementation approach suggested by our findings is to consider how larger clinics may serve as models for smaller clinics, specifically with regard to organizational characteristics that may be normatively linked to but not necessarily dependent on clinic size and infrastructure. For example, a close examination of our results on the first dimension reveals that the two variables most highly associated with reach include interaction between clinicians and staff and level of standardization involved in administration of our DA. Our findings show that larger clinics (with greater patient volume and number of evaluations) have greater communication and standardization of clinic procedures. This may be because, just as standardization in industry allows for economies of scale and enables markets to optimize their transactional efficiency, it is also likely that greater standardization and greater organizational guidance and oversight from physicians are features that help larger clinics to efficiently and effectively (safely) deliver services to large patient populations, particularly where staff members may be busiest and most time-constrained [38] While these structural and organizational features may be more prevalent among larger, highly resourced clinics, they are not dependent on clinic size and may serve as goalposts for smaller clinics hoping to effectively position themselves for successful implementation.

To better understand the significance of these organizational variables and their "active ingredients" for implementation success, we turn to a growing literature in implementation science and clinical decision support. Specifically, what may account for the positive impacts of frequent interaction between clinicians and staff, and what might these interactions entail? Studies from over 
two decades of implementation research suggest that implementation success is typically bolstered by having at least one clinician champion to promote the use of a decision support intervention [24, 33, 39-43]. Interactions that demonstrate support and endorsement of a decision support tool from a respected clinician (especially a physician) "messenger" can offer the extra incentive needed for a clinical staff member to prioritize and recognize the value of an intervention [37]. The critical role of clinician champions has also recently been highlighted by Berry et al. [42], who found that designating a clinical lead for implementation helped to address staff misunderstandings about which contexts and resources were best suited for administering decisional support. Brinkman et al. [43] likewise found that implementation was facilitated by buy-in from physicians about the value of SDM and formal training workshops for clinical staff implementing decision support. Further, a study by Uy et al. [16] similarly identified physician support as "crucial" to the distribution success of patient decision supports. In our own experience, we observed that communication between and among clinicians and coordinators offered important opportunities to communicate buy-in from clinicians to coordinators and other members of the clinical team who prioritized use of our DA as a result.

In our implementation project, we observed that physician buy-in was most crucial at the first stages of implementation (orientation and startup) to demonstrate support of our DA from clinical leaders and to provide the leverage necessary to make workflow changes or transition towards integration with or (in rarer cases) replacement of existing patient education materials. After this initial startup period, clinicians were called on less often to actively demonstrate their support as coordinators increasingly sustained their own momentum and expertise in using the DA. Thus, we recommend enlisting clinician support at early stages of implementation to leverage their practical knowledge and influence.

The studies cited above indicate that frequent clinician and clinical staff interaction may positively impact on implementation success by providing incentives and motivations for clinical staff engagement. However, because we did not see this variable appear alongside the attitudinal and motivational dimension revealed by our PCA, we must consider that other aspects of interaction may be equally important. In particular, interaction between clinical team members may also help to communicate practical support and guidelines for how to undergo implementation in ways that are consistent with site-specific goals and available resources. Tietbohl et al. [44] showed that clinical sites with high implementation success exhibited frequent, timely and accurate communication between clinicians and clinical staff, while lower performing clinics had more contentious relationships and inadequate communication. The nature of these interactions involved conveying practical guidance, troubleshooting and ongoing feedback to keep staff apprised of their distribution progress. Interaction may thus be associated with greater standardization because interaction provides a forum for communicating practical, concrete steps towards standardizing use of an intervention in practice. Cuypers et al. [45] similarly found that success in implementing a decision support tool was dependent on integrating clinical team members not only to influence their motivation but also to help navigate the clinical infrastructure needed to integrate and standardize use of an intervention into daily work patterns.

The importance of making an intervention "visible" in routine practice can also enhance use of decision support and systematize implementation by providing reminders to use the tool, encouraging strategic placement of visual cues in the workspace such as distribution checklists on computers, providing pre-written scripts with talking points for staff administering the DA, and scheduling timely feedback sessions for staff to discuss implementation progress [17]. In a further example from our own project, one of our highest-reach sites used our DA as part of a more extensive clinical evaluation and patient education checklist instituted and championed by the director of the LVAD program. Other examples from the literature (e.g. Scalia et al. [33]) suggest that standardizing use of a decision support tool as part of a mandate or milestone completion expected by a clinical supervisor can result in improved interactions between patients and healthcare professionals, with patients asking more questions and feeling more satisfied and empowered in decision making. Evidence suggests that interaction with clinician champions can also provide structural insights about how best to systematize procedures within existing flow to facilitate referral, ordering and administration of DAs to patients [10, 24, 33, 42].

Examples of how to effectively standardize or systematize implementation to promote use of a DA include systematically identifying eligible patients to receive decision support tools in advance of their clinical visits [33], offering referral or ordering options in a patient's electronic health record to ensure availability of a DA for patients to review ahead of their clinic visits [43], and having a DA readily accessible at a standardized place and time of decision making [24]. Based on our results and experience, contextualized by these previous studies, we thus offer an additional concrete recommendation to standardize the time, place and process for administering decision support using checklists or "kits" to ensure their 
availability, salience and convenience for routine use, particularly in fast-paced, busy clinical settings.

\section{The importance of multi-directional communication}

We also observed that physicians are not always the only individuals to conceive of or initiate pro-implementation changes, and that the direction of effective communication is not always "top-down" (i.e. physician to coordinator) but multidirectional. Physicians can also learn from coordinators, nurses and other clinical staff who work in more regular proximity with patients about other "ground-level" considerations that are important for administering decision supports effectively or meaningfully in the daily clinic setting. In our own project, we observed that certain LVAD coordinators were the de facto champions of using our DA in practice and were effective at generating awareness and support from clinicians and other clinical staff. Coordinators harbor a wealth of observational experience that inform practical suggestions and solutions for implementation success at the patient level. Thus, while physician champions may be more suited to authorize and gain higher-level buyin for infrastructural changes, bi-directional communication with nurse coordinators and other clinical staff can help to generate "grass roots" support and practical insights for implementing an intervention effectively in routine practice. We believe that our findings offer further support for the importance of fostering effective communication channels between physicians and staff in order to integrate different and equally important perspectives on engaging stakeholders at multiple levels (administrative, clinician-, staff- and patient-level). Based on these insights, we thus offer a final recommendation: to create forums for frequent exchange of perspectives and practical information across multiple roles, prioritizing "on the ground" insights (e.g. from coordinators) within a larger context of organizational resources and constraints (conveyed by clinicians or administrative personnel). The recommendations discussed above are listed in Table 7 as key takeaways for researchers interested in empirical insights to inform implementation science theory, and/or for clinicians and clinical programs seeking to better position themselves for implementation success.

\section{Limitations}

A primary limitation is that our analysis leaves over $50 \%$ of the variance unexplained by the measured variables. Further, the proportion of variance among variables that might be due to common variance, was moderately low (0.38), indicating that the variables we measured may be only loosely related and require further verification, or a larger number of cases to better understand variable associations.
Table 7 Summary of recommendations for decision support implementation

Generate staff interest in and motivation to use SDM tools

Enlist clinician support at early stages of implementation to leverage their practical knowledge and influence

Standardize the time, place and process for administering decision support

Create forums for frequent exchange of perspectives and practical information across multiple roles

A second limitation is that we measured "interaction between clinicians and staff" by asking respondents to report on frequency of interaction. Further, we asked about "level of standardization" with reference to timing consistency - that is, whether coordinators' administered the DA at the same time in the educational process across patients. A potential shortcoming of these phrasings is that other aspects of interaction and standardization beyond frequency and timing, respectively, may be equally or more important. Greater insights are needed into which features of clinician-coordinator interaction and standardization impact on implementation success.

A third limitation is that our results are based on respondents' perceptions and may thus not accurately reflect the actual clinic characteristics and team-wide attitudes. Further research involving a more extensive range of clinics as well as rigorous measurement of site characteristics is needed to confirm our findings.

\section{Conclusion}

A critical goal among implementation researchers and policy makers is to identify clinical site characteristics that facilitate implementation success of a clinical intervention. Our study highlights two distinct groups of site characteristics empirically associated with greater use of a decision support tool and suggests that successful implementation plans should incorporate specific efforts to promote supportive and mutually informative interactions between clinical staff members and to institute systematic and standardized protocols to enhance the availability, convenience and salience of intervention tool in routine practice. Our results provide insights that are supported by a growing implementation science literature and may be useful for clinicians and LVAD programs seeking to better position themselves for effective integration of decision support into their patient education, or to evaluate how existing site dynamics might forecast certain implementation outcomes.

\section{Abbreviations}

DA: Decision Aid; D\&l: Dissemination and Implementation; ITS: Implementation tracking sheet; LVAD: Left ventricular assist device; PCA: Principal 
components analysis; RCT: Randomized Controlled Trial; SDM: Shared decision-making.

\section{Acknowledgements}

Not applicable

\section{Authors' contributions}

MT collected the data for this analysis. AB, AC, JG, DH, UJ, ML, MM, NS, VT, $\mathrm{BT}, \mathrm{NU}$ and JE facilitated data collection at their clinical sites and provided feedback on drafts of the manuscript. KK analyzed the data, interpreted findings and was a primary contributor in writing the manuscript. KK and JBB conceptualized the manuscript's focus and methodology. RV and JE provided substantive feedback for revisions. All authors read and approved this manuscript.

\section{Funding}

Findings reported in this publication were funded through a Patient-Centered Outcomes Research Institute (PCORI) Award (DI-2017C2-7726). The statements in this publication are solely the responsibility of the authors and do not necessarily represent the views of the Patient-Centered Outcomes Research Institute (PCORI), its Board of Governors or Methodology Committee.

\section{Availability of data and materials}

The datasets used and/or analyzed during the current study are available from the corresponding author on reasonable request.

\section{Declarations}

\section{Ethics approval and consent to participate}

Not applicable. Under 45 CFR 46.102(I), quality improvement activities limited to collecting provider data regarding the implementation of a practice do not satisfy the definition of research. As such, there is no requirement for these activities to be conducted with informed consent, and there is no requirement for these activities to be reviewed by an IRB.

\section{Consent for publication}

Not applicable.

\section{Competing interests}

The authors have no competing interests to declare.

\begin{abstract}
Author details
${ }^{1}$ Center for Medical Ethics and Health Policy, Baylor College of Medicine, One Baylor Plaza MC: 420, Houston, TX 77030, USA. ${ }^{2}$ Division of Heart Failure, Houston Methodist Hospital, Smith Tower, 6550 Fannin St., Ste 1901, Houston, TX 77030, USA. ${ }^{3}$ Baylor St. Luke's Medical Center, Texas Heart Institute, 7200 Cambridge Street, Ste 6C, Houston, TX 77030, USA. ${ }^{4}$ Duchossois Center for Advanced Medicine - Hyde Park, University of Chicago Medicine, 5758 S. Maryland Ave., Chicago, IL 60637, USA. ${ }^{5}$ INTREGIS Advanced Cardiac Care, 3400 N.W. Expressway, Bldg C. Suite 200, Oklahoma City, OK 73112, USA. ${ }^{6}$ Division of Cardiology, Montefiore Medical Center, Bronx, NY 10467, USA. ${ }^{7}$ Miami Transplant Institute, University of Miami Health System, Miami, FL 33136, USA. ${ }^{8}$ Cardiovascular Medicine, Brigham and Women's Hospital, 75 Francis St. Boston, MA 02115, USA. ${ }^{9}$ Aurora St. Luke's Medical Center, 2900 W Oklahoma Ave, Milwaukee, WI 53215, USA. ${ }^{10}$ Asheville Cardiology Associates, 5 Vanderbilt Park Dr., Asheville, NC 28803, USA. ${ }^{11}$ Columbia Presbyterian Medical Center, Columbia University Irving Medical Center, 622 West 168th St., Room 129, New York, NY 10032, USA. ${ }^{12}$ Department of Health Services Research, Division of Cancer Prevention and Population Services, University of Texas MD Anderson Cancer Center, 1515 Holcombe Blvd., Unit 1465, Houston, TX, USA. ${ }^{13}$ Miller Family Heart and Vascular Institute, Cleveland Clinic, 9500 Euclid Ave., Cleveland, $\mathrm{OH}$ 44195, USA.
\end{abstract}

Received: 9 November 2020 Accepted: 10 March 2021 Published online: 20 March 2021

\section{References}

1. Scholl I, LaRussa A, Hahlweg P, Kobrin S, Elwyn G. Organizational- and system-level characteristics that influence implementation of shared decision-making and strategies to address them: a scoping review. Implement Sci. 2018 Mar 9;13(1):40.

2. Damschroder LJ, Aron DC, Keith RE, Kirsh SR, Alexander JA, Lowery JC. Fostering implementation of health services research findings into practice: a consolidated framework for advancing implementation science. Implement Sci. 2009 Aug;7(4):50.

3. Chaudoir SR, Dugan AG, Barr CH. Measuring factors affecting implementation of health innovations: a systematic review of structural, organizational, provider, patient, and innovation level measures. Implement Sci. 2013 Feb 17:8(1):22.

4. Michie S, van Stralen MM, West R. The behaviour change wheel: a new method for characterising and designing behaviour change interventions. Implement Sci. 2011 Apr 23;6(1):42.

5. Atkins L, Francis J, Islam R, O'Connor D, Patey A, Ivers N, et al. A guide to using the Theoretical Domains Framework of behaviour change to investigate implementation problems. Implement Sci. 2017 Jun 21;12(1):77.

6. Cane J, O'Connor D, Michie S. Validation of the theoretical domains framework for use in behaviour change and implementation research. Implement Sci. 2012 Apr 24;7(1):37.

7. Elwyn G, Scholl I, Tietbohl C, Mann M, Edwards AGK, Clay C, et al. "Many miles to go ...": a systematic review of the implementation of patient decision support interventions into routine clinical practice. BMC Med Inform Decis Mak. 2013;13(Suppl 2):S14.

8. Gayer CC, Crowley MJ, Lawrence WF, Gierisch JM, Gaglio B, Williams JW, et al. An overview and discussion of the Patient-Centered Outcomes Research Institute's decision aid portfolio. J Comp Effect Res. 2016 Jun 14;5(4):407-15.

9. Stacey D, Légaré F, Lewis K, Barry MJ, Bennett CL, Eden KB, et al. Decision aids for people facing health treatment or screening decisions. Cochrane Database of Systematic Reviews [Internet]. 2017 [cited 2020 Jul 20];(4). https://www.cochranelibrary.com/cdsr/doi/https://doi.org/10.1002/ 14651858.CD001431.pub5/full

10. Stacey D, Suwalska V, Boland L, Lewis KB, Presseau J, Thomson R. Are patient decision aids used in clinical practice after rigorous evaluation? A survey of trial authors. Med Decis Making. 2019 Oct 1;39(7):805-15.

11. Holmes-Rovner M, Valade D, Orlowski C, Draus C, Nabozny-Valerio B, Keiser S. Implementing shared decision-making in routine practice: barriers and opportunities. Health Expect. 2000 Sep;3(3):182-91.

12. Greenhalgh T, Robert G, Macfarlane F, Bate P, Kyriakidou O. Diffusion of innovations in service organizations: systematic review and recommendations. Milbank Q. 2004 Dec;82(4):581-629.

13. Légaré F, Ratté S, Gravel K, Graham ID. Barriers and facilitators to implementing shared decision-making in clinical practice: update of a systematic review of health professionals' perceptions. Patient Educ Couns. 2008 Dec;73(3):526-35.

14. Feibelmann S, Yang TS, Uzogara EE, Sepucha K. What does it take to have sustained use of decision aids? A programme evaluation for the Breast Cancer Initiative. Health Expect. 2011;14(s1):85-95.

15. Sepucha KR, Simmons LH, Barry MJ, Edgman-Levitan S, Licurse AM, Chaguturu SK. Ten years, forty decision aids, and thousands of patient uses: shared decision making at Massachusetts general Hospital. Health Aff (Millwood). 2016 Apr;35(4):630-6.

16. Uy V, May SG, Tietbohl C, Frosch DL. Barriers and facilitators to routine distribution of patient decision support interventions: a preliminary study in community-based primary care settings. Health Expect. 2014 Jun;17(3):353-64.

17. Tietbohl CK, Rendle KAS, Halley MC, May SG, Lin GA, Frosch DL. Implementation of patient decision support interventions in primary care: the role of relational coordination. Med Decis Making. 2015;35(8):987-98.

18. Blumenthal-Barby JS, Kostick KM, Delgado ED, Volk RJ, Kaplan HM, Wilhelms LA, et al. Assessment of patients' and caregivers' informational and decisional needs for left ventricular assist device placement: implications for informed consent and shared decision-making. J Heart Lung Transpl. 2015 Sep;34(9):1182-9.

19. Kostick K, Delgado E, Wilhelms L, Bruce C, Estep J, Loebe M, et al. Development and Pilot-Testing of a Patient Decision Aid for Left Ventricular Assist Device Placement. The VAD Journal [Internet]. 2016 Feb 4;2(1). https://uknowledge.uky.edu/vad/vol2/iss1/1 
20. Kostick KM, Minard CG, Wilhelms LA, Delgado E, Abraham M, Bruce CR, et al. Development and validation of a patient-centered knowledge scale for left ventricular assist device placement. J Heart Lung Transpl. 2016 Jun 1;35(6):768-76.

21. Kostick KM, Bruce CR, Minard CG, Volk RJ, Civitello A, Krim SR, et al. A multisite randomized controlled trial of a patient-centered ventricular assist device decision aid (VADDA Trial). J Card Fail. 2018 Oct;24(10):661-71.

22. Elwyn G, Durand MA, Song J, Aarts J, Barr PJ, Berger Z, et al. A three-talk model for shared decision making: multistage consultation process. BMJ [Internet]. 2017 Nov 6 [cited 2020 Jul 21];359. https://www.bmj.com/ content/359/bmj.j4891

23. Glasgow RE, Vogt TM, Boles SM. Evaluating the public health impact of health promotion interventions: the RE-AIM framework. Am J Public Health. 1999 Sep;89(9):1322-7.

24. Hsu C, Liss DT, Westbrook EO, Arterburn D. Incorporating patient decision aids into standard clinical practice in an integrated delivery system: medical decision making [Internet]. 2013 Jan 8 [cited 2020 Jul 24]; https:// journals.sagepub.com/doi/https://doi.org/10.1177/0272989X12468615

25. Stacey D, Vandemheen KL, Hennessey R, Gooyers T, Gaudet E, Mallick R, et al. Implementation of a cystic fibrosis lung transplant referral patient decision aid in routine clinical practice: an observational study. Implement Sci. 2015 Feb 7;10(1):17.

26. van Tol-Geerdink JJ, van Oort IM, Somford DM, Wijburg CJ, Geboers A, van Uden-Kraan CF, et al. Implementation of a decision aid for localized prostate cancer in routine care: a successful implementation strategy. Health Inf J. 2020 Jun 1;26(2):1194-207.

27. Shea CM, Jacobs SR, Esserman DA, Bruce K, Weiner BJ. Organizational readiness for implementing change: a psychometric assessment of a new measure. Implement Sci. 2014 Jan 10;9(1):7.

28. Gagnon M-P, Desmartis M, Labrecque M, Car J, Pagliari C, Pluye P, et al. Systematic review of factors influencing the adoption of information and communication technologies by healthcare professionals. J Med Syst. 2012 Feb;36(1):241-77.

29. Elwyn G, Taubert M, Kowalczuk J. Sticky knowledge: a possible model for investigating implementation in healthcare contexts. Implement Sci. 2007 Dec 20;2(1):44

30. Moullin JC, Sabater-Hernández D, Fernandez-Llimos F, Benrimoj SI. A systematic review of implementation frameworks of innovations in healthcare and resulting generic implementation framework. Health Res Policy Syst. 2015 Mar 14;13(1):16.

31. Colón-Emeric C, Toles M, Cary MP, Batchelor-Murphy M, Yap T, Song $Y$, et al. Sustaining complex interventions in long-term care: a qualitative study of direct care staff and managers. Implement Sci. $2016 \mathrm{Jul}$ 16;11(1):94

32. Waltz TJ, Powell BJ, Matthieu MM, Damschroder L, Chinman MJ, Smith $J \mathrm{~L}$, et al. Use of concept mapping to characterize relationships among implementation strategies and assess their feasibility and importance: results from the Expert Recommendations for Implementing Change
(ERIC) study. Implement Sci [Internet]. 2015 Aug 7 [cited 2020 Jul 28];10. https://www.ncbi.nlm.nih.gov/pmc/articles/PMC4527340/

33. Scalia P, Elwyn G, Durand M-A. Provoking conversations": case studies of organizations where Option $\mathrm{Grid}^{\mathrm{TM}}$ decision aids have become'normalized. BMC Med Inform Decis Mak. 2017 Aug 18;17(1):124.

34. Nilsen P, Roback K, Broström A, Ellström P-E. Creatures of habit: accounting for the role of habit in implementation research on clinical behaviour change. Implement Sci. 2012 Jun 9;7(1):53.

35. Gagné ME, Boulet L-P. Implementation of asthma clinical practice guidelines in primary care: a cross-sectional study based on the Knowledge-toAction Cycle. J Asthma. 2018 Mar 4;55(3):310-7.

36. Simon HA. Administrative Behavior, 4th Edition. 4th ed. Simon and Schuster; $1997.390 \mathrm{p}$

37. Kostick KM, Trejo M, Volk RJ, Estep JD, Blumenthal-Barby JS. Using nudges to enhance clinicians'implementation of shared decision making with patient decision aids. MDM Policy Pract. 2020 Jan 1;5(1):2381468320915906

38. Tassey G. Standardization in technology-based markets. Res Policy. 2000 Apr 1;29(4):587-602.

39. Holmes-Rovner M, Kelly-Blake K, Dwamena F, Dontje K, Henry RC, Olomu $A$, et al. Shared decision making guidance reminders in practice (SDMGRIP). Patient Educ Couns. 2011 Nov;85(2):219-24.

40. Belkora JK, Teng A, Volz S, Loth MK, Esserman L. Expanding the reach of decision and communication aids in a breast care center: a quality improvement study. Patient Educ Couns. 2011 May;83(2):234-9.

41. Lewis $\mathrm{CL}$, Pignone MP. Promoting informed decision-making in a primary care practice by implementing decision aids. N C Med J. 2009; 70(2):136-9

42. Berry DL, Hong F, Halpenny B, Sanda MG, Master VA, Filson CP, et al. Evaluating clinical implementation approaches for prostate cancer decision support. Urology Practice. 2019 Mar 1;6(2):93-9.

43. Brinkman WB, Lipstein EA, Taylor J, Schoettker PJ, Naylor K, Jones K, et al. Design and implementation of a decision aid for juvenile idiopathic arthritis medication choices. Pediatric Rheumatol. 2017 Jun 5;15(1):48.

44. Tietbohl CK, Rendle KAS, Halley MC, May SG, Lin GA, Frosch DL. Implementation of patient decision support interventions in primary care: the role of relational coordination. Med Decis Making. 2015 Nov 1;35(8):987-98.

45. Cuypers M, Al-Itejawi HHM, van Uden-Kraan CF, Stalmeier PFM, Lamers RED, van Oort IM, Aids ID, into Routine Prostate Cancer Care in The Netherlands: Implementation and Patient Evaluations from the Multi-regional JIPPA Initiative.J Canc Educ [Internet]. et al. cited 2020 Jul 20. 2019 Jul 5. https://doi.org/10.1007/s13187-019-01572-9.

\section{Publisher's Note}

Springer Nature remains neutral with regard to jurisdictional claims in published maps and institutional affiliations.
Ready to submit your research? Choose BMC and benefit from:

- fast, convenient online submission

- thorough peer review by experienced researchers in your field

- rapid publication on acceptance

- support for research data, including large and complex data types

- gold Open Access which fosters wider collaboration and increased citations

- maximum visibility for your research: over 100M website views per year

At BMC, research is always in progress.

Learn more biomedcentral.com/submissions 